PROCEEDINGS OF THE

AMERICAN MATHEMATICAL SOCIETY

Volume 131, Number 12, Pages 3795-3806

S 0002-9939(03)06946-6

Article electronically published on March 25, 2003

\title{
A REPRODUCING KERNEL SPACE MODEL FOR $\mathbf{N}_{\kappa}$-FUNCTIONS
}

\author{
VLADIMIR DERKACH AND SEPPO HASSI
}

(Communicated by Joseph A. Ball)

\begin{abstract}
A new model for generalized Nevanlinna functions $Q \in \mathbf{N}_{\kappa}$ will be presented. It involves Bezoutians and companion operators associated with certain polynomials determined by the generalized zeros and poles of $Q$. The model is obtained by coupling two operator models and expressed by means of abstract boundary mappings and the corresponding Weyl functions.
\end{abstract}

\section{INTRODUCTION}

A function $Q$, locally meromorphic on $\mathbb{C} \backslash \mathbb{R}$, belongs to the class $\mathbf{N}_{\kappa}\left(\kappa \in \mathbb{Z}_{+}\right)$ of generalized Nevanlinna functions if on its domain of holomorphy $\rho(Q)$ it admits the symmetry property $Q(\bar{z})=\overline{Q(z)}$, and is such that the Nevanlinna kernel

$$
\mathrm{N}_{Q}(z, w)=\frac{Q(z)-Q(\bar{w})}{z-\bar{w}}, z \neq \bar{w}, \quad \mathrm{~N}_{Q}(z, \bar{z})=Q^{\prime}(z)
$$

$z, w \in \rho(Q)$, has $\kappa$ negative squares; cf. [16]. As is known [17, every generalized Nevanlinna function $Q \in \mathbf{N}_{\kappa}$ holomorphic at $i \in \rho(Q)$ admits the representation

$$
Q(z)=s+z[v, v]+\left(z^{2}+1\right)\left[(A-z)^{-1} v, v\right], \quad s=\bar{s},
$$

with a selfadjoint operator (or a linear relation) $A$ acting in a Hilbert space $(\mathfrak{H},[\cdot, \cdot])$ and a generating vector $v \in \mathfrak{H}$, and then $Q$ is called the $Q$-function of a symmetric restriction $S$ of $A$. Models for Pontryagin space selfadjoint operators has been constructed in [16] and [15]. In particular, the model in [15], which is an extension of the one given in [14] for the case $\kappa=1$, was based on the use of certain distributions to define a Pontryagin space $\Pi_{\kappa}$, as a finite dimensional extension of a Hilbert space $L_{2}(\sigma)$, and a selfadjoint multiplication operator in $\Pi_{\kappa}$.

In the present paper a new and explicit model for the functions $Q \in \mathbf{N}_{\kappa}$ will be given. This model uses a recent factorization result from [10] which states that every function $Q \in \mathbf{N}_{\kappa}$ admits a representation

$$
Q=r^{\sharp} Q_{0} r, \quad r^{\sharp}(z)=\overline{r(\bar{z})},
$$

where $r=\frac{p}{q}$ is a rational function and $Q_{0}$ is a Nevanlinna function $\left(Q_{0} \in \mathbf{N}_{0}\right)$. The model is constructed as a certain coupling of a Hilbert space selfadjoint operator

Received by the editors December 7, 2001 and, in revised form, July 10, 2002.

2000 Mathematics Subject Classification. Primary 47B25, 47B50; Secondary 46C20, 46E22.

Key words and phrases. Generalized Nevanlinna function, symmetric operator, selfadjoint extension, Weyl function, boundary triplet, reproducing kernel Pontryagin space.

The support of the Academy of Finland (projects 40362 and 52528) is gratefully acknowledged. 
$A_{0}$ generated by $Q_{0}$ in (1.3) and a selfadjoint operator $A_{R}$ acting on a finitedimensional Pontryagin space $\mathfrak{H}_{R}$. The inner product in $\mathfrak{H}_{R}$ is defined with the aid of the Bezoutian $B_{p, q}$ associated with the polynomials $p, q$, and the operator $A_{R}$ is the orthogonal sum $C_{p^{\sharp}} \oplus C_{p}$ of the companion matrices determined by $p, p^{\sharp}$.

The main tools of the construction are the technique of the reproducing kernel Pontryagin spaces (see e.g. [1, [21]) and the boundary value approach developed in [13], [8], [2], 3]. In particular, some results obtained in [3] for orthogonal couplings of symmetric operators, which are described in terms of abstract boundary conditions, play an important role in the proof of Theorem 3.3. The models constructed in [5], 6] for giving realizations for singular perturbations of selfadjoint operators (cf. e.g. [11]) can be seen as special cases of the present model.

The use of abstract boundary mappings makes it easy to apply the present model, for instance, to spectral problems for differential operators with rationally $\lambda$ depending boundary conditions. These problems were shown to be adequate to the eigenvalue problems for some Pontryagin space selfadjoint operators; see [20], [12. In [7] (see also [6]) a special case of the present model where $r$ is a polynomial has been applied to give realizations for singular perturbations of selfadjoint (differential) operators.

\section{Preliminaries}

Let $\mathfrak{H}$ be a separable Pontryagin space, let $S$ be a not necessarily densely defined closed symmetric relation in $\mathfrak{H}$ with equal defect numbers $d=d_{+}(S)=d_{-}(S)$ $(<\infty)$, and let $S^{*}$ be the adjoint linear relation of $S$. A triplet $\Pi=\left\{\mathcal{H}, \Gamma_{0}, \Gamma_{1}\right\}$, where $\mathcal{H}$ is a Hilbert space and $\Gamma_{j}, j=0,1$, are linear mappings from $S^{*}$ to $\mathcal{H}$, is said to be a boundary triplet for $S^{*}$, if the mapping $\Gamma=\left(\Gamma_{0}, \Gamma_{1}\right)^{\top}: \widehat{f} \rightarrow\left\{\Gamma_{0} \widehat{f}, \Gamma_{1} \widehat{f}\right\}$ from $S^{*}$ into $\mathcal{H} \oplus \mathcal{H}$ is surjective and the abstract Green's identity

$$
\left(f^{\prime}, g\right)-\left(f, g^{\prime}\right)=\left(\Gamma_{1} \widehat{f}, \Gamma_{0} \widehat{g}\right)_{\mathcal{H}}-\left(\Gamma_{0} \widehat{f}, \Gamma_{1} \widehat{g}\right)_{\mathcal{H}}
$$

holds for all $\widehat{f}=\left\{f, f^{\prime}\right\}, \widehat{g}=\left\{g, g^{\prime}\right\} \in S^{*}$; cf. [13], [8], [2]. The mapping $\Gamma$ from $S^{*}$ onto $\mathcal{H} \oplus \mathcal{H}$ establishes a one-to-one correspondence between the set $\operatorname{Ext}_{S}$ of all closed extensions of $S$ and the set $\widetilde{\mathcal{C}}(\mathcal{H})$ of all closed linear relations in $\mathcal{H}$ via

$$
\widetilde{A}_{\theta}:=\Gamma^{-1} \theta=\left\{\widehat{f} \in S^{*}: \Gamma \widehat{f} \in \theta\right\}, \quad \theta \in \widetilde{\mathcal{C}}(\mathcal{H}) .
$$

It follows from (2.1) that

$$
\widetilde{A}_{\theta}^{*}=\widetilde{A}_{\theta^{*}}, \quad \text { for every } \theta \in \widetilde{\mathcal{C}}(\mathcal{H}) .
$$

As usual $A_{0}$ and $A_{1}$ stand for the selfadjoint extensions $A_{j}=\operatorname{ker} \Gamma_{j}, j=0,1$. Let $\mathfrak{N}_{\lambda}\left(S^{*}\right)=\operatorname{ker}\left(S^{*}-\lambda\right)$ be a defect subspace of $S$ and let $\widehat{\mathfrak{N}}_{\lambda}:=\left\{\left\{f_{\lambda}, \lambda f_{\lambda}\right\}: f_{\lambda} \in\right.$ $\left.\mathfrak{N}_{\lambda}\left(S^{*}\right)\right\}$. The $\gamma$-field and the Weyl function associated with $\Pi$ are defined by

$$
\gamma(\lambda)=p_{1}\left(\Gamma_{0} \mid \widehat{\mathfrak{N}}_{\lambda}\right)^{-1}\left(\in\left[\mathcal{H}, \mathfrak{N}_{\lambda}\right]\right), \quad Q(\lambda)=\Gamma_{1}\left(\Gamma_{0}\left\lceil\widehat{\mathfrak{N}}_{\lambda}\right)^{-1}(\in[\mathcal{H}]),\right.
$$

$\lambda \in \rho\left(A_{0}\right) \neq \emptyset$ (see [8], [2]). In (2.4) $p_{1}$ denotes the orthogonal projection onto the first component of $\mathcal{H} \oplus \mathcal{H}$. The operator-valued functions $\gamma$ and $Q$ are holomorphic on $\rho\left(A_{0}\right)$. The set of all points of regular type of $S$ will be denoted by $\widehat{\rho}(S)$. A closed symmetric relation $S$ is said to be simple if

$$
\mathfrak{H}=\overline{\operatorname{span}}\{\gamma(z): z \in \widehat{\rho}(S) \supset \mathbb{C} \backslash \mathbb{R}\} .
$$


In this case, the number $\kappa$ of negative squares of the kernel $\mathrm{N}_{Q}$ in (1.1) coincides with the dimension of a maximal negative subspace of $\mathfrak{H}$. The class of Weyl functions $Q$ of $S$ coincides with the class of $Q$-functions of $S$ in the sense of [16].

Associated with the kernel $\mathrm{N}_{Q}$ is a reproducing kernel Pontryagin space $\mathfrak{H}(Q)$ of analytic vector functions on $\rho(Q)$ (cf. [1], 21]) generated by the vector functions $z \rightarrow \mathrm{N}_{Q}(z, w) h, w \in \rho(Q), h \in \mathcal{H}$, and the inner product

$$
\left[\mathrm{N}_{Q}(\cdot, w) h, \mathrm{~N}_{Q}(\cdot, z) k\right]=k^{*} \mathrm{~N}_{Q}(z, w) h, \quad h, k \in \mathcal{H}, \quad z, w \in \rho(Q) .
$$

The characterizing property of $\mathfrak{H}(Q)$ is the equality

$$
\left[F(\cdot), \mathrm{N}_{Q}(\cdot, w) k\right]=k^{*} F(w), \quad f \in \mathfrak{H}(Q), \quad k \in \mathcal{H}, \quad w \in \rho(Q) .
$$

The multiplication operator $S(Q)$ in $\mathfrak{H}(Q)$ defined by

$$
S(Q)=\left\{\left\{f, f^{\prime}\right\} \in \mathfrak{H}(Q)^{2}: f^{\prime}(w)=w f(w), w \in \rho(Q)\right\}
$$

is symmetric. The next proposition specifies its adjoint $S(Q)^{*}$ and associates a boundary triplet to $S(Q)^{*}$, such that $Q$ is the corresponding Weyl function.

Proposition 2.1 (9], [4). Let $Q \in \mathbf{N}_{\kappa}$ be a Weyl function in $\mathcal{H}=\mathbb{C}^{d}$ of a closed symmetric operator $S$ in a Pontryagin space and let $\mathfrak{H}(Q)$ be the reproducing kernel Pontryagin space associated with the kernel (1.1). Then $S(Q)$ in (2.8) is a closed simple symmetric operator in $\mathfrak{H}(Q)$ (which is unitarily equivalent to $S$ if $S$ is simple) and, moreover:

(i) the adjoint $S(Q)^{*}$ is given by

$$
S(Q)^{*}=\left\{\widehat{F}=\left\{F, F^{\prime}\right\} \in \mathfrak{H}(Q)^{2}: F^{\prime}(w)-w F(w)=\xi_{1}-Q(w) \xi_{0}, \xi_{0}, \xi_{1} \in \mathcal{H}\right\} ;
$$

(ii) the boundary triplet $\left\{\mathcal{H}, \Gamma_{0}, \Gamma_{1}\right\}$ for $S(Q)^{*}$ is given by

$$
\mathcal{H}=\mathbb{C}^{d}, \quad \Gamma_{0} \widehat{F}=\xi_{0}, \quad \Gamma_{1} \widehat{F}=\xi_{1} ;
$$

(iii) the corresponding Weyl function coincides with $Q$ and the $\gamma$-field is given by

$$
\gamma_{Q}(z)=\mathrm{N}_{Q}(\cdot, \bar{z}), \quad z \in \rho(Q) .
$$

\section{An operator model associated With the Class $\mathbf{N}_{\kappa}$}

Let $p$ and $q$ be scalar polynomials with complex coefficients of the form $\left(p_{n} \neq 0\right)$

$$
p(\lambda)=p_{n} \lambda^{n}+p_{n-1} \lambda^{n-1}+\cdots+p_{0}, \quad q(\lambda)=q_{n} \lambda^{n}+q_{n-1} \lambda^{n-1}+\cdots+q_{0} .
$$

Then the Bezoutian Bez $(p, q)$ and the companion matrix $C_{p}$ are defined by

$$
\frac{p(\ell) q(\lambda)-q(\ell) p(\lambda)}{\ell-\lambda}=L \operatorname{Bez}(p, q) \Lambda^{\top}, \quad C_{p}=\left(\begin{array}{ccccc}
0 & 1 & 0 & \ldots & 0 \\
0 & 0 & 1 & \ddots & \vdots \\
\vdots & & \ddots & \ddots & 0 \\
0 & & & 0 & 1 \\
-\widetilde{p}_{0} & -\widetilde{p}_{1} & \ldots & \ldots & -\widetilde{p}_{n-1}
\end{array}\right) \text {, }
$$

with $\Lambda_{(n)}=\left(I_{\mathcal{H}}, \lambda I_{\mathcal{H}}, \ldots, \lambda^{n-1} I_{\mathcal{H}}\right), L_{(n)}=\left(I_{\mathcal{H}}, \ell I_{\mathcal{H}}, \ldots, \ell^{n-1} I_{\mathcal{H}}\right)$, and $\widetilde{p}_{i}=p_{n}^{-1} p_{i}$, $i=0,1, \ldots, n-1$. The following facts are needed in the sequel (cf. e.g. [19]):

(i) $B_{p}=\operatorname{Bez}(p, 1)=\left[p_{i+j+1}\right]_{i, j=0}^{n-1}\left(p_{j}=0\right.$ if $\left.j>n\right)$;

(ii) $\operatorname{Bez}\left(p, \lambda^{k}\right)=B_{p} C_{p}^{k}$;

(iii) $\operatorname{Bez}(p, q)=\sum_{j=0}^{n} q_{j} \operatorname{Bez}\left(p, \lambda^{j}\right)=B_{p} q\left(C_{p}\right)$ (the Barnett factorization); 
(iv) $B_{p} C_{p}=C_{p}^{\top} B_{p}$;

(v) $\sigma\left(C_{p}\right)=\sigma(p)$ (the set of zeros of $p$ ).

In particular, (iii) shows that $\operatorname{Bez}(p, q)$ is invertible if and only if $q\left(C_{p}\right)$ is invertible, which holds precisely when $\sigma\left(C_{p}\right)$ does not contain zeros of $q$, or equivalently, that $p$ and $q$ are relatively prime, i.e., $\sigma(p) \cap \sigma(q)=\emptyset$. The items (iii) and (iv) show that $\operatorname{Bez}(p, q)^{\top}=\operatorname{Bez}(p, q)$ and $\operatorname{Bez}(p, q) C_{p}=C_{p}^{\top} \operatorname{Bez}(p, q)$. The statement in (v) can be augmented with the corresponding root vectors. For each $\lambda_{j} \in \sigma\left(C_{p}\right)$ with multiplicity $\kappa_{j}$ one associates the set of Vandermonde vectors,

$$
V_{k}\left(\lambda_{j}\right)=\left.\frac{1}{k !} \frac{d^{k}}{d \lambda^{k}} \Lambda\right|_{\lambda=\lambda_{j}}, \quad k=0,1, \ldots, \kappa_{j},
$$

which form a full chain of the root subspace $\operatorname{ker}\left(C_{p}-\lambda_{j}\right)^{\kappa_{j}}$. In what follows $\left(e_{j}\right)_{j=1}^{n}$ stands for the standard basis in $\mathbb{C}^{n}$. It is easy to check that

$$
\begin{aligned}
w: & =\operatorname{Bez}(p, q) e_{n}=\sum_{j=0}^{n-1} p_{n} q_{j} B_{p} C_{p}^{j} e_{n}+q_{n} B_{p} C_{p}^{n} e_{n} \\
& =\left(p_{n} q_{0}-q_{n} p_{0}, p_{n} q_{1}-q_{n} p_{1}, \ldots, p_{n} q_{n-1}-q_{n} p_{n-1}\right)^{\top} .
\end{aligned}
$$

Now introduce the rational functions $r, r^{\sharp}$, and $R$ by

$$
r=\frac{p}{q}, \quad r^{\sharp}=\frac{p^{\sharp}}{q^{\sharp}}, \quad R=\left(\begin{array}{cc}
0 & r \\
r^{\sharp} & 0
\end{array}\right),
$$

where $p^{\sharp}(z)=\overline{p(\bar{z})}$ and $q^{\sharp}(z)=\overline{q(\bar{z})}$. Then $R$ is a matrix function which belongs to the class $\mathbf{N}_{\kappa}$ with $\kappa=\max \{\operatorname{deg} p, \operatorname{deg} q\}$. The next result gives an explicit form for the reproducing kernel space $\mathfrak{H}(R)$ associated with $R$.

Proposition 3.1. Let the polynomials $p$ and $q$ in (3.1) be relatively prime and assume that $\operatorname{deg} p \geq \operatorname{deg} q$ and that $p$ is monic. Let $w$ and $R$ be given by (3.3) and (3.4), respectively. Then:

(i) The reproducing kernel Pontryagin space $\mathfrak{H}(R)$ is isometrically isomorphic to the space $\mathfrak{H}_{R}=\mathbb{C}^{n} \oplus \mathbb{C}^{n}$ equipped with the inner product

$$
[\cdot, \cdot]_{\mathfrak{H}_{R}}=(\mathcal{B} \cdot, \cdot), \quad \mathcal{B}=\left(\begin{array}{cc}
0 & B_{p, q} \\
B_{p, q}^{*} & 0
\end{array}\right), \quad B_{p, q}=\operatorname{Bez}(p, q) .
$$

(ii) The restriction $S_{R}$ of $\mathcal{C}_{p}=C_{p^{\sharp}} \oplus C_{p}$ to the domain

$$
\operatorname{dom} S_{R}=\left\{F=f \oplus \tilde{f} \in \mathfrak{H}_{R}: \bar{w}^{*} \tilde{f}=w^{*} f=0\right\}
$$

is a simple symmetric operator in $\mathfrak{H}_{R}$, which is unitarily equivalent to $S(R)$.

(iii) The adjoint $S_{R}^{*}$ of $S_{R}$ takes the form

$$
S_{R}^{*}=\left\{\widehat{F}=\left\{F, \mathcal{C}_{p} F+\left(\widetilde{c} e_{n}, c e_{n}\right)^{\top}\right\}: F=f \oplus \widetilde{f} \in \mathfrak{H}_{R}, \quad c, \widetilde{c} \in \mathbb{C}\right\} .
$$

(iv) A boundary triplet $\Pi_{R}=\left\{\mathbb{C}^{2}, \Gamma_{0}^{R}, \Gamma_{1}^{R}\right\}$ for $S_{R}^{*}$ can be defined by

$$
\Gamma_{0}^{R} \widehat{F}=\left(\begin{array}{l}
\bar{q}_{n} \widetilde{c}+w^{*} f \\
q_{n} c+\bar{w}^{*} \widetilde{f}
\end{array}\right), \quad \Gamma_{1}^{R} \widehat{F}=\left(\begin{array}{c}
c \\
\widetilde{c}
\end{array}\right), \quad \widehat{F} \in S_{R}^{*} .
$$

(v) The corresponding $\gamma$-field is given by $\gamma_{R}(\lambda)=1 / q^{\sharp}(\lambda) \Lambda^{\top} \oplus 1 / q(\lambda) \Lambda^{\top}$ and the Weyl function $M_{R}$ coincides with $R$ in (3.4). 
Proof. The kernel $\mathrm{N}_{r}(\ell, \lambda)$ can be expressed in terms of $B_{p, q}$ as follows:

$$
\mathrm{N}_{r}(\ell, \lambda)=\frac{1}{q(\ell)} L B_{p, q} \Lambda^{*} \frac{1}{q(\bar{\lambda})} .
$$

This leads to the following factorization for the kernel $\mathrm{N}_{R}(\ell, \lambda)$ :

$$
\mathrm{N}_{R}(\ell, \lambda)=\left(\begin{array}{cc}
\frac{1}{q(\ell)} L & 0 \\
0 & \frac{1}{q^{\sharp}(\ell)} L
\end{array}\right)\left(\begin{array}{cc}
0 & B_{p, q} \\
B_{p, q}^{*} & 0
\end{array}\right)\left(\begin{array}{cc}
\frac{1}{q(\lambda)} \Lambda & 0 \\
0 & \frac{1}{q^{\sharp}(\lambda)} \Lambda
\end{array}\right)^{*} .
$$

Thus, $R$ is a matrix Nevanlinna function with $n$ negative (and $n$ positive) squares.

(i) It follows from the factorization (3.9) that $\mathfrak{H}(R)$ consists of vector functions

$$
F(\ell)=\left(\begin{array}{cc}
\frac{1}{q(\ell)} L & 0 \\
0 & \frac{1}{q^{\sharp}(\ell)} L
\end{array}\right) \mathcal{B} F, \quad F=\left(\begin{array}{l}
f \\
\widetilde{f}
\end{array}\right) \in \mathfrak{H}_{R} .
$$

Now the identities $[F(\cdot), G(\cdot)]_{\mathfrak{H}(R)}=G^{*} \mathcal{B} F=[F, G]_{\mathfrak{H}_{R}}$ show that the mapping $F \rightarrow F(\cdot)$ determines an isometric isomorphism between $\mathfrak{H}_{R}$ and $\mathfrak{H}(R)$.

(ii) By the properties given above $\left(\mathcal{B C}_{p}\right)^{*}=\mathcal{B C}_{p}$, i.e., $\mathcal{C}_{p}$ is selfadjoint in $\mathfrak{H}_{R}$. It is clear from (3.6) that $S_{R}$ is a closed symmetric operator in $\mathfrak{H}_{R}$ with defect numbers $(2,2)$. For every $K=k \oplus \widetilde{k} \in \mathfrak{H}_{R}$ the following equalities are easily checked:

$$
\left\{\begin{aligned}
\lambda \Lambda B_{p} \widetilde{k} & =\Lambda B_{p} C_{p} \widetilde{k}+p(\lambda) \widetilde{k}_{1}, \\
\lambda \Lambda B_{p}^{*} k & =\Lambda B_{p}^{*} C_{p^{\sharp}} k+p^{\sharp}(\lambda) k_{1} .
\end{aligned}\right.
$$

Moreover,

$$
\left(q\left(C_{p}\right) \widetilde{f}\right)_{1}=q_{n}\left(-p_{0} \widetilde{f}_{1}-\cdots-p_{n-1} \widetilde{f}_{n}\right)+q_{n-1} \widetilde{f}_{n}+\cdots+q_{0} \widetilde{f}_{1}=\bar{w}^{*} \widetilde{f}
$$

and similarly $\left(q^{\sharp}\left(C_{p^{\sharp}}\right) f\right)_{1}=w^{*} f$. Therefore, applying the identities (3.11) with $\widetilde{k}=q\left(C_{p}\right) \tilde{f}, k=q^{\sharp}\left(C_{p^{\sharp}}\right) f$ and taking into account the Barnett factorization, one arrives at the following decomposition for the function $\lambda F(\lambda)$ :

$$
\lambda F(\lambda)=\left(\begin{array}{cc}
\frac{1}{q(\lambda)} \Lambda & 0 \\
0 & \frac{1}{q^{\sharp}(\lambda)} \Lambda
\end{array}\right) \mathcal{B C}_{p}\left(\begin{array}{l}
f \\
\widetilde{f}
\end{array}\right)+R(\lambda)\left(\begin{array}{l}
w^{*} f \\
\bar{w}^{*} \widetilde{f}
\end{array}\right) .
$$

This implies the unitary equivalence of the operators $S_{R}$ and $S(R)$.

(iii) Denote by $T$ the set on the right-hand side of (3.7) and assume that $\{F, G\} \in$ $T$. Then the selfadjointness of $\mathcal{C}_{p}$ in $\mathfrak{H}_{R}$ shows that for all $\{H, K\} \in S_{R}$,

$$
[G, H]_{\mathfrak{H}_{R}}-[F, K]_{\mathfrak{H}_{R}}=\left[\left(\begin{array}{c}
\widetilde{c} e_{n} \\
c e_{n}
\end{array}\right),\left(\begin{array}{l}
k \\
\widetilde{k}
\end{array}\right)\right]_{\mathfrak{H}_{R}}=\left(\mathcal{B}\left(\begin{array}{c}
\widetilde{c} e_{n} \\
c e_{n}
\end{array}\right),\left(\begin{array}{l}
k \\
\widetilde{k}
\end{array}\right)\right)=\left(\begin{array}{l}
c k^{*} w \\
\widetilde{c} k^{*} \bar{w}
\end{array}\right)=0
$$

cf. (3.3). Thus, $T \subset S_{R}^{*}$ and the equality (3.7) follows by a dimension argument.

(iv) \& (v) Since $p_{n}=1$ one can write $\Lambda w=q(\lambda)-q_{n} p(\lambda)$ and this leads to

$$
\frac{1}{q(\lambda)} \Lambda w=1-q_{n} r(\lambda), \quad \frac{1}{q^{\sharp}(\lambda)} \Lambda \bar{w}=1-\bar{q}_{n} r^{\sharp}(\lambda) .
$$


Therefore, with $\widehat{F}=\{F, G\} \in S_{R}^{*}$ one obtains from (3.3), (3.7), and (3.12)

$$
\begin{aligned}
G(\lambda)-\lambda F(\lambda) & =\left(\begin{array}{cc}
\frac{1}{q(\lambda)} \Lambda & 0 \\
0 & \frac{1}{q^{\sharp}(\lambda)} \Lambda
\end{array}\right) \mathcal{B}\left(G-\mathcal{C}_{p} F\right)-R(\lambda)\left(\begin{array}{l}
w^{*} f \\
\bar{w}^{*} \widetilde{f}
\end{array}\right) \\
& =\left(\begin{array}{c}
\frac{c}{q(\lambda)} \Lambda w \\
\frac{\widetilde{c}}{q^{\sharp}(\lambda)} \Lambda \bar{w}
\end{array}\right)-R(\lambda)\left(\begin{array}{l}
w^{*} f \\
\bar{w}^{*} \widetilde{f}
\end{array}\right)=\left(\begin{array}{l}
c \\
\widetilde{c}
\end{array}\right)-R(\lambda)\left(\begin{array}{l}
\bar{q}_{n} \widetilde{c}+w^{*} f \\
q_{n} c+\bar{w}^{*} \widetilde{f}
\end{array}\right) .
\end{aligned}
$$

Now, it follows from Proposition 2.1 that $\Gamma_{0}^{R}$ and $\Gamma_{1}^{R}$ as defined in (iv) can be taken to be the boundary operators for $S_{R}^{*}$ and that $R$ is the corresponding Weyl function. The form of the $\gamma$-field follows from (2.9), (3.9), and (3.10).

Corollary 3.2. Let the assumptions be as in Proposition 3.1. Then:

(i) The selfadjoint extension $A_{1}^{R}=\operatorname{ker} \Gamma_{1}^{R}$ of $S_{R}$ is an operator which coincides with $\mathcal{C}_{p}=C_{p^{\sharp}} \oplus C_{p}$.

(ii) The selfadjoint extension $A_{0}^{R}=\operatorname{ker} \Gamma_{0}^{R}$ of $S_{R}$ is an operator if and only if $\operatorname{deg} p=\operatorname{deg} q$, in which case it coincides with $\mathcal{C}_{q}=C_{q^{\sharp}} \oplus C_{q}$.

Proof. The statement (i) is obtained by taking $c=\widetilde{c}=0$ in part (iii) of Proposition 3.1. To see (ii) first assume that $\operatorname{deg} q<\operatorname{deg} p$. Then $q_{n}=0$ and it follows from the formulas for $S_{R}^{*}$ and $\Gamma_{0}^{R}$ in Proposition 3.1 that mul $A_{0}^{R}$ is nontrivial. Next assume that $\operatorname{deg} q=\operatorname{deg} p$. Then $q_{n} \neq 0$ and $\widehat{F} \in A_{0}^{R}$ implies that

$$
c=-\frac{1}{q_{n}} \bar{w}^{*} \widetilde{f}, \quad \widetilde{c}=-\frac{1}{\bar{q}_{n}} w^{*} f .
$$

Observe that $w / q_{n}=\left(C_{p}^{\top}-C_{q}^{\top}\right) e_{n}$. Substituting this and (3.13) into (3.7) yields

$$
\begin{aligned}
& C_{p^{\sharp}} f-e_{n} \frac{1}{\bar{q}_{n}} w^{*} f=C_{p^{\sharp}} f-e_{n} e_{n}^{\top}\left(C_{p^{\sharp}}-C_{q^{\sharp}}\right) f=C_{p^{\sharp}} f-\left(C_{p^{\sharp}}-C_{q^{\sharp}}\right) f=C_{q^{\sharp}} f, \\
& C_{p} \widetilde{f}-e_{n} \frac{1}{q_{n}} \bar{w}^{*} \widetilde{f}=C_{p} \widetilde{f}-e_{n} e_{n}^{\top}\left(C_{p}-C_{q}\right) \widetilde{f}=C_{p} \widetilde{f}-\left(C_{p}-C_{q}\right) \widetilde{f}=C_{q} \widetilde{f},
\end{aligned}
$$

which proves (ii).

The construction of the model for $\mathbf{N}_{\kappa}$-functions is based on the following theorem. An underlying idea here arises from some results on intermediate extensions that were proved in 3 ; cf. also [5] for the case of Pontryagin spaces. Given two symmetric operators $S_{1}$ and $S_{2}$ with the Weyl functions $Q_{1}$ and $Q=\left(Q_{i j}\right)_{i, j=1}^{2}, Q$ decomposed according to $\mathcal{H}=\mathcal{H}_{1} \oplus \mathcal{H}_{2}$, one produces two intermediate extensions whose Weyl functions are of the form

$$
\begin{aligned}
& \widetilde{Q}(\lambda)=Q_{1}(\lambda) \pi_{1}+Q(\lambda)=\left(\begin{array}{cc}
Q_{1}(\lambda)+Q_{11}(\lambda) & Q_{12}(\lambda) \\
Q_{21}(\lambda) & Q_{22}(\lambda)
\end{array}\right), \\
& \widetilde{Q}_{2}(\lambda)=\left(\pi_{2} Q(\lambda)^{-1}\left\lceil\mathcal{H}_{2}\right)^{-1}=Q_{22}(\lambda)-Q_{21}(\lambda) Q_{11}^{-1}(\lambda) Q_{12}(\lambda),\right.
\end{aligned}
$$

where $\pi_{j}$ stands for the orthogonal projection onto $\mathcal{H}_{j}, j=1,2$, and then combines these two transforms by applying (3.15) to (3.14). All of this can be shortly expressed by using abstract boundary conditions. The procedure is applied here to a scalar function $Q_{1}=-M_{0}^{-1}$ and the matrix function $Q=R$ in Proposition 3.1. 
Theorem 3.3. Let the polynomials $p$ and $q$ in (3.1) be relatively prime, let $r$ and $R$ be given by (3.4), and let $S_{R}$ be the symmetric operator in $\mathfrak{H}_{R}$ as defined in Proposition [3.1] with the boundary triplet $\Pi_{R}=\left\{\mathbb{C}^{2}, \Gamma_{0}^{R}, \Gamma_{1}^{R}\right\}$. Let $S_{0}$ be a symmetric operator in the Hilbert space $\mathfrak{H}_{0}=\mathfrak{H}\left(M_{0}\right)$ corresponding to the Weyl function $M_{0}$ of the boundary triplet $\Pi_{0}=\left\{\mathbb{C}, \Gamma_{0}^{0}, \Gamma_{1}^{0}\right\}$ of $S_{0}^{*}$, and let $w$ be given by (3.3). Denote $\widehat{F}=\left\{F, F^{\prime}\right\}$ with $F=f \oplus \widetilde{f} \in \mathfrak{H}_{R}$ and let $\widehat{f}_{0}=\left\{f_{0}, f_{0}^{\prime}\right\} \in S_{0}^{*}$. Then:

(i) The linear relation

$$
S=\left\{\left\{f_{0} \oplus F, f_{0}^{\prime} \oplus\left(\mathcal{C}_{p} F+\left(0, \Gamma_{0}^{0} \widehat{f}_{0} e_{n}\right)^{\top}\right)\right\}: \bar{w}^{*} \widetilde{f}=-q_{n} \Gamma_{0}^{0} \widehat{f}_{0}, w^{*} f=\Gamma_{1}^{0} \widehat{f}_{0}\right\}
$$

is closed and symmetric in $\mathfrak{H}=\mathfrak{H}_{0} \oplus \mathfrak{H}_{R}$.

(ii) The adjoint $S^{*}$ of $S$ is given by

$S^{*}=\left\{\left\{f_{0} \oplus F, f_{0}^{\prime} \oplus\left(\mathcal{C}_{p} F+\left(\widetilde{c} e_{n}, \Gamma_{0}^{0} \widehat{f}_{0} e_{n}\right)^{\top}\right)\right\}: \bar{q}_{n} \widetilde{c}+w^{*} f=\Gamma_{1}^{0} \widehat{f_{0}}, \widetilde{c} \in \mathbb{C}\right\}$.

(iii) A boundary triplet $\Pi=\left\{\mathbb{C}, \Gamma_{0}, \Gamma_{1}\right\}$ for $S^{*}$ is determined by

$$
\Gamma_{0}\left(\widehat{f}_{0} \oplus \widehat{F}\right)=q_{n} \Gamma_{0}^{0} \widehat{f}_{0}+\bar{w}^{*} \widetilde{f}, \quad \Gamma_{1}\left(\widehat{f_{0}} \oplus \widehat{F}\right)=\widetilde{c}, \quad \widehat{f_{0}} \oplus \widehat{F} \in S^{*} .
$$

(iv) The corresponding $\gamma$-field and the Weyl function are given by

$$
\begin{gathered}
\gamma(\lambda)=\gamma_{0}(\lambda) r(\lambda) \oplus\left(\frac{r(\lambda) M_{0}(\lambda)}{q^{\sharp}(\lambda)} \Lambda^{\top} \dot{+} \frac{1}{q(\lambda)} \Lambda^{\top}\right), \\
M(\lambda)=r^{\sharp}(\lambda) M_{0}(\lambda) r(\lambda) .
\end{gathered}
$$

Proof. Step 1. Define an intermediate extension $\widetilde{S}$ of $S_{0} \oplus S_{R}$ by

$$
\widetilde{S}=\left\{\widehat{f}_{0} \oplus \widehat{F} \in S_{0}^{*} \oplus S_{R}^{*}: \Gamma_{1}^{0} \widehat{f}_{0}=\Gamma_{0}^{R} \widehat{F}=\left(\Gamma_{0}^{0} \widehat{f}_{0}\right) e_{1}-\Gamma_{1}^{R} \widehat{F}=0\right\},
$$

where $e_{1}=(1,0)^{\top} \in \mathbb{C}^{2}$. Then $\widetilde{S}$ is closed and symmetric. Using (2.3) it can be seen that the adjoint $\widetilde{S}^{*}$ of $\widetilde{S}$ is of the form (cf. also [5, Proposition 2.3])

$$
\widetilde{S}^{*}=\left\{\widehat{f_{0}} \oplus \widehat{F} \in S_{0}^{*} \oplus S_{R}^{*}: \Gamma_{1}^{0} \widehat{f}_{0}-\pi_{1} \Gamma_{0}^{R} \widehat{F}=0\right\},
$$

where $\pi_{1}=e_{1} e_{1}^{*}$. Moreover, one can take $\widetilde{\Pi}=\left\{\mathcal{H}_{0} \oplus \mathcal{H}_{0}, \Gamma_{0}^{R},\left(-\Gamma_{0}^{0}\right) e_{1}+\Gamma_{1}^{R}\right\}$ to be a boundary triplet for $\widetilde{S}^{*}$. The corresponding Weyl function is given by

$$
\widetilde{M}=\left(\begin{array}{cc}
-M_{0}^{-1} & r \\
r^{\sharp} & 0
\end{array}\right) \text {. }
$$

Step 2. Next define a closed symmetric extension $S$ of $\widetilde{S}$ in (3.18) via

$$
\begin{aligned}
S & =\left\{\widehat{f_{0}} \oplus \widehat{F} \in \widetilde{S}^{*}: \pi_{2} \Gamma_{0}^{R} \widehat{F}=\left(\Gamma_{0}^{0} \widehat{f}_{0}\right) e_{1}-\Gamma_{1}^{R} \widehat{F}=0\right\} \\
& =\left\{\widehat{f_{0}} \oplus \widehat{F} \in S_{0}^{*} \oplus S_{R}^{*}: \Gamma_{1}^{0} \widehat{f}_{0}=\bar{q}_{n} \widetilde{c}+w^{*} f, \Gamma_{0}^{0} \widehat{f}_{0}=c, q_{n} c+\bar{w}^{*} \widetilde{f}=\widetilde{c}=0\right\},
\end{aligned}
$$

where $\pi_{2}=I-\pi_{1}$. In view of (3.7) $S$ can be rewritten as in (i). Moreover,

$$
\begin{aligned}
S^{*} & =\left\{\widehat{f_{0}} \oplus \widehat{F} \in \widetilde{S}^{*}: \Gamma_{0}^{0} \widehat{f}_{0}-\pi_{1} \Gamma_{1}^{R} \widehat{F}=0\right\} \\
& =\left\{\widehat{f_{0}} \oplus \widehat{F} \in S_{0}^{*} \oplus S_{R}^{*}: \Gamma_{1}^{0} \widehat{f}_{0}=\bar{q}_{n} \widetilde{c}+w^{*} f, \Gamma_{0}^{0} \widehat{f}_{0}=c\right\},
\end{aligned}
$$

which leads to (ii). A boundary triplet for $S^{*}$ is obtained by letting

$$
\Gamma_{0} \widehat{f}=\pi_{2} \Gamma_{0}^{R} \widehat{F}=q_{n} \Gamma_{0}^{0} \widehat{f}_{0}+\bar{w}^{*} \widetilde{f}, \quad \Gamma_{1} \widehat{f}=\pi_{2}\left(\left(-\Gamma_{0}^{0} \widehat{f_{0}}\right) e_{1}+\Gamma_{1}^{R} \widehat{F}\right)=\pi_{2} \Gamma_{1}^{R} \widehat{F}=\widetilde{c},
$$


which gives (iii) (cf. [5, Proposition 2.2]). Finally, to prove (iv) observe that the defect subspace $\mathfrak{N}_{\lambda}\left(S^{*}\right)$ consists of vectors

$$
f_{0} \oplus F=\gamma_{0}(\lambda) \xi \oplus\left(\begin{array}{cc}
\frac{1}{q^{\sharp}(\lambda)} \Lambda^{\top} & 0 \\
0 & \frac{1}{q(\lambda)} \Lambda^{\top}
\end{array}\right)\left(\begin{array}{l}
h_{1} \\
h_{2}
\end{array}\right), \quad \xi, h_{1}, h_{2} \in \mathbb{C},
$$

such that $\widehat{f}_{0, \lambda}=\left\{f_{0}, \lambda f_{0}\right\} \in S_{0}^{*}$ and $\widehat{F}_{\lambda}=\{F, \lambda F\} \in S_{R}^{*}$ satisfy the equalities

$$
\Gamma_{1}^{0} \widehat{f}_{0, \lambda}-\pi_{1} \Gamma_{0}^{R} \widehat{F}_{\lambda}=\Gamma_{0}^{0} \widehat{f}_{0, \lambda}-\pi_{1} \Gamma_{1}^{R} \widehat{F}_{\lambda}=0 ;
$$

cf. (3.19), (3.21). One can rewrite (3.22) in the form

$$
\xi-r(\lambda) h_{2}=M_{0}(\lambda) \xi-h_{1}=0 .
$$

Therefore, $\mathfrak{N}_{\lambda}\left(S^{*}\right)$ is spanned by the vectors

$$
\gamma(\lambda) h_{2}=\gamma_{0}(\lambda) r(\lambda) h_{2} \oplus\left(\begin{array}{cc}
\frac{1}{q^{\sharp}(\lambda)} \Lambda^{\top} & 0 \\
0 & \frac{1}{q(\lambda)} \Lambda^{\top}
\end{array}\right)\left(\begin{array}{c}
M_{0}(\lambda) r(\lambda) h_{2} \\
h_{2}
\end{array}\right), \quad h_{2} \in \mathbb{C},
$$

and this gives (3.16). Similarly, (3.17) follows from $\Gamma_{1}\left(\widehat{f}_{0, \lambda} \oplus \widehat{F}_{\lambda}\right)=\pi_{2} R(\lambda) h=$ $r^{\sharp}(\lambda) M_{0}(\lambda) r(\lambda) h_{2}$. Notice that the same expression for $M$ is also obtained by applying the transform (3.15) to (3.20).

To explain the importance of Theorem 3.3 let $M$ be a generalized Nevanlinna function in $\mathbf{N}_{\kappa}$. Let $\alpha_{j}$ be all the poles in $\mathbb{C}^{+}$and the generalized poles of nonpositive type in $\mathbb{R}$ of $M$ with multiplicities $\kappa_{j}, j=1, \ldots, t$, and let $\beta_{i}$ be all the zeros in $\mathbb{C}_{+}$and the generalized zeros of nonpositive type in $\mathbb{R}$ of $M$ with multiplicities $\pi_{i}, i=1, \ldots, s$; see [18]. Define the polynomials $p$ and $q$ as follows:

$$
p(z)=\prod_{j=1}^{s}\left(z-\beta_{j}\right)^{\pi_{j}}, \quad q(z)=\prod_{i=1}^{t}\left(z-\alpha_{i}\right)^{\kappa_{i}} .
$$

The factorization result in [10] when applied to $M \not \equiv 0$ shows that there exists a (unique) Nevanlinna function $M_{0} \in \mathbf{N}_{0}$, i.e. $\kappa=0$, such that (3.17) holds with $r=p / q$. The converse is also true. If $p$ and $q$ are relatively prime polynomials, and if $M_{0} \in \mathbf{N}_{0}, M_{0} \not \equiv 0$, then $M$ in (3.17) belongs to $\mathbf{N}_{\kappa}$, with $\kappa=\max \{\operatorname{deg} p, \operatorname{deg} q\}$. Moreover, the zeros of $p$ and $q$ coincide, counting multiplicities, with the finite (generalized) poles and zeros of nonpositive type of $M$, and $\operatorname{deg} q-\operatorname{deg} p=\kappa_{\infty}-\pi_{\infty}$; cf. [4, Proposition 3.2]. Now Theorem 3.3 applied to the polynomials $p$ and $q$ gives a model for $M$ as a coupling of the finite-dimensional model for $R$ in Proposition 3.1 with $r=p / q$, and a Hilbert space model for $M_{0}$. In fact, the approach for constructing the model via Theorem 3.3 allows one to apply the same method immediately to factorized matrix Nevanlinna functions for which the corresponding Bezoutian is invertible.

\section{Simplicity OF THE MOdel OPERATOR}

The symmetric linear relation $S$ in Theorem 3.3 need not be an operator. In fact, from the form of $S$ it is seen that if $\operatorname{deg} p=\operatorname{deg} q$, then mul $S=\operatorname{mul} S_{0} \oplus\{0\} \oplus\{0\}$, 
while if $\operatorname{deg} p>\operatorname{deg} q$, then

$$
\operatorname{mul} S=\left\{f_{0}^{\prime} \oplus\left(\begin{array}{c}
0 \\
\Gamma_{0}^{0} \widehat{f_{0}} e_{n}
\end{array}\right): \widehat{f}_{0}=\left\{0, f_{0}^{\prime}\right\} \in A_{1}=\operatorname{ker} \Gamma_{1}^{0}\right\} .
$$

Hence, if $\operatorname{deg} p=\operatorname{deg} q$, then $S$ is an operator if and only if $S_{0}$ is an operator and if $\operatorname{deg} p>\operatorname{deg} q$, then $S$ is an operator if and only if $A_{1}$ is an operator. A simple symmetric relation is necessarily an operator. The next theorem characterizes the simplicity of $S$ in spectral theoretical terms.

Theorem 4.1. Let the symmetric operator $S_{0}$ in $\mathfrak{H}_{0}$ be simple and let $S$ be as defined in Theorem 3.3 with relatively prime polynomials $p$ and $q$ as in (3.1). Then:

(i) If $\operatorname{deg} p=\operatorname{deg} q$, then $S$ is simple if and only if

$$
\sigma_{p}\left(A_{0}\right) \cap \sigma(p)=\emptyset \text { and } \sigma_{p}\left(A_{1}\right) \cap \sigma(q)=\emptyset .
$$

(ii) If $\operatorname{deg} p>\operatorname{deg} q$, then $S$ is simple if and only if $A_{1}=\operatorname{ker} \Gamma_{1}^{0}$ is an operator and the conditions (4.2) are satisfied.

(iii) If $\operatorname{deg} p<\operatorname{deg} q$, then $S$ is simple if and only if $A_{0}=\operatorname{ker} \Gamma_{0}^{0}$ is an operator and the conditions (4.2) are satisfied.

Moreover, the $\gamma$-fields $\gamma$ and $\gamma_{1}$ of $H_{0}=\operatorname{ker} \Gamma_{0}$ and $H_{1}=\operatorname{ker} \Gamma_{1}$ have the expansions

$$
\begin{aligned}
q(\lambda) \gamma(\lambda) & =\sum_{k=0}^{\pi_{i}-1}\left(\lambda-\beta_{i}\right)^{k} \Gamma_{k, i}+\left(\lambda-\beta_{i}\right)^{\pi_{i}-1} o(1), \lambda \widehat{\rightarrow} \beta_{i} \in \sigma(p) \backslash \sigma_{p}\left(A_{0}\right), \\
p^{\sharp}(\lambda) \gamma_{1}(\lambda) & =\sum_{k=0}^{\kappa_{j}-1}\left(\lambda-\bar{\alpha}_{j}\right)^{k} \widetilde{\Gamma}_{k, j}+\left(\lambda-\bar{\alpha}_{j}\right)^{\kappa_{j}-1} o(1), \bar{\lambda} \widehat{\rightarrow} \alpha_{j} \in \sigma(q) \backslash \sigma_{p}\left(A_{1}\right),
\end{aligned}
$$

$i=1, \ldots, s, j=1, \ldots, t$, where $\Gamma_{k, i}=0 \oplus 0 \oplus V_{k}\left(\beta_{i}\right), \widetilde{\Gamma}_{k, j}=0 \oplus V_{k}\left(\bar{\alpha}_{j}\right) \oplus 0$, and $V_{k}\left(\beta_{i}\right), V_{k}\left(\bar{\alpha}_{j}\right)$ are the corresponding Vandermonde vectors.

Proof. First the expansions (4.3) and (4.4) are derived. For every $\beta_{i} \in \sigma(p) \backslash \sigma_{p}\left(A_{0}\right)$ the $\gamma$-field $\gamma_{0}$ and the Weyl function $M_{0}$ corresponding to $A_{0}$ satisfy the relations

$$
\lim _{\lambda \rightarrow \beta_{i}}\left(\lambda-\beta_{i}\right) \gamma_{0}(\lambda)=\lim _{\lambda \rightarrow \beta_{i}}\left(\lambda-\beta_{i}\right) M_{0}(\lambda)=0 .
$$

By incorporating the Taylor series for the vector function $\Lambda$ at $\lambda=\beta_{i}$ in the expression for the $\gamma$-field in (3.16) and taking into account (4.5), one obtains the expansion (4.3) with $\beta_{i} \in \sigma(p) \backslash \sigma_{p}\left(A_{0}\right)$ of multiplicity $\pi_{i}, i=1, \ldots, s$. The $\gamma$-field $\gamma_{1}$ and the Weyl function $M_{1}$ corresponding to $H_{1}=\operatorname{ker} \Gamma_{1}$ are given by

$$
\gamma_{1}=\gamma M^{-1}, \quad M_{1}=-M^{-1}=-\frac{q q^{\sharp}}{p p^{\sharp}} M_{0}^{-1} .
$$

The $\gamma$-field $\gamma_{0} M_{0}^{-1}$ and the Weyl function $-M_{0}^{-1}$ corresponding to $A_{1}$ satisfy

$$
\lim _{\lambda \leadsto \bar{\alpha}_{j}}\left(\lambda-\bar{\alpha}_{j}\right) \gamma_{0}(\lambda) M_{0}(\lambda)^{-1}=\lim _{\lambda \leftrightharpoons \bar{\alpha}_{j}}\left(\lambda-\bar{\alpha}_{j}\right) M_{0}(\lambda)^{-1}=0
$$

for every $\alpha_{j} \in \sigma(q) \backslash \sigma_{p}\left(A_{1}\right)$. It follows from (3.16) that for every $\lambda \in \rho\left(A_{1}\right) \backslash \sigma(p)$,

$$
p^{\sharp}(\lambda) \gamma_{1}(\lambda)=\gamma_{0}(\lambda) \frac{q^{\sharp}(\lambda)}{M_{0}(\lambda)} \oplus\left(\Lambda^{\top} \dot{+} \Lambda^{\top} \frac{q^{\sharp}(\lambda)}{p(\lambda) M_{0}(\lambda)}\right) .
$$

Hence, by incorporating the Taylor series for $\Lambda$ at $\lambda=\bar{\alpha}_{j}$ in (4.8) and using (4.7) one obtains the expansion (4.4) with $\alpha_{j} \in \sigma(q) \backslash \sigma_{p}\left(A_{1}\right)$ of multiplicity $\kappa_{j}, j=1, \ldots, t$. 
Proof of sufficiency. Let $\widetilde{\mathfrak{H}}=\overline{\operatorname{span}}\left\{\mathfrak{N}_{\lambda}\left(S^{*}\right): \lambda \in \rho\left(A_{0}\right)\right\}$. Then $\widetilde{\mathfrak{H}} \subset \mathfrak{H}$ and the simplicity of $S$ follows by proving $\mathfrak{H} \subset \widetilde{\mathfrak{H}}$. The expansions (4.3) and (4.4) imply

$$
\begin{aligned}
& \Gamma_{k, i} \in \widetilde{\mathfrak{H}}, k=1, \ldots, \pi_{i}, \quad i=1, \ldots, s, \\
& \widetilde{\Gamma}_{k, j} \in \widetilde{\mathfrak{H}}, \quad k=1, \ldots, \kappa_{j}, \quad j=1, \ldots, t .
\end{aligned}
$$

If $\operatorname{deg} p=n$, the Vandermonde vectors $V_{k}\left(\beta_{i}\right), k=1, \ldots, \pi_{i}, i=1, \ldots, s$, span $\mathbb{C}^{n}$, and (4.9) gives $\{0\} \oplus\{0\} \oplus \mathbb{C}^{n} \subset \widetilde{\mathfrak{H}}$. Similarly, if $\operatorname{deg} q=n$, the Vandermonde vectors $V_{k}\left(\bar{\alpha}_{j}\right), k=1, \ldots, \kappa_{j}, j=1, \ldots, t$, span $\mathbb{C}^{n}$, and (4.10) gives $\{0\} \oplus \mathbb{C}^{n} \oplus\{0\} \subset \widetilde{\mathfrak{H}}$. To treat the case $\operatorname{deg} p \neq \operatorname{deg} q$ one may assume that $n=\operatorname{deg} p>\operatorname{deg} q=m$. Then by the assumptions $A_{1}$ is an operator, which implies that

$$
\lim _{\lambda \rightrightarrows \infty} \gamma_{0}(\lambda) M_{0}(\lambda)^{-1}=0, \quad \lim _{\lambda \rightarrow \infty} \frac{1}{\lambda} M_{0}(\lambda)^{-1}=0 .
$$

Hence, it follows from (4.8) that

$$
\lim _{\lambda \leftrightharpoons \infty} \frac{p^{\sharp}(\lambda) \gamma_{1}(\lambda)}{\lambda^{n-1}}=0 \oplus e_{n} \oplus 0 .
$$

Analogously, for $k=1, \ldots, n-m-1$ one obtains

$$
\lim _{\lambda \rightarrow \infty} \lambda^{k}\left(\frac{p^{\sharp}(\lambda) \gamma_{1}(\lambda)}{\lambda^{n-1}}-0 \oplus \sum_{j=0}^{k-1} \frac{e_{n-j}}{\lambda^{j}} \oplus 0\right)=0 \oplus e_{n-k} \oplus 0 .
$$

This together with (4.10) implies that $\{0\} \oplus \mathbb{C}^{n} \oplus\{0\} \subset \widetilde{\mathfrak{H}}$, since the first $m$ coordinates of the Vandermonde vectors $V_{k}\left(\bar{\alpha}_{j}\right), k=1, \ldots, \kappa_{j}$, form the full chain of the root subspace of the $m \times m$ companion matrix $C_{q^{\sharp}}$ at $\bar{\alpha}_{j} \in \sigma\left(C_{q^{\sharp}}\right), j=$ $1, \ldots, t$. The simplicity of $S_{0}$ and the form of the $\gamma$-field in (3.16) finally show that $\mathfrak{H}_{0} \oplus\{0\} \oplus\{0\} \subset \widetilde{\mathfrak{H}}$. Therefore, $\mathfrak{H} \subset \widetilde{\mathfrak{H}}$ and $S$ is simple.

Proof of necessity. If $\operatorname{deg} p>\operatorname{deg} q$ and mul $A_{1} \neq\{0\}$, then also mul $S \neq\{0\}$, which is not possible if $S$ is simple. Similarly, if $\operatorname{deg} p<\operatorname{deg} q$, then the simplicity of $S$ forces that $A_{0}$ is an operator. It remains to prove the necessity of the conditions (4.2). First assume that $\beta_{i} \in \sigma_{p}\left(A_{0}\right) \cap \sigma(p)$. Let $h_{i} \neq 0$ be the corresponding eigenvector of $A_{0}$ and let $\widehat{h}_{i}=\left\{h_{i}, \beta_{i} h_{i}\right\}$. Then $\beta_{i} \in \mathbb{R}$ and $\Gamma_{0}^{0} \widehat{h}_{i}=0$. Let $\widehat{v}_{i}=\left\{v_{i}, \beta_{i} v_{i}\right\}$, where

$$
v_{i}=\frac{q^{\sharp}\left(\beta_{i}\right)}{\Gamma_{1}^{0} \widehat{h}_{i}} h_{i} \oplus\left(\begin{array}{c}
V_{0}\left(\beta_{i}\right) \\
0
\end{array}\right) .
$$

Here $q^{\sharp}\left(\beta_{i}\right) \neq 0$, since $\sigma(p) \cap \sigma(q)=\emptyset$, and $\Gamma_{1}^{0} \widehat{h}_{i} \neq 0$ since $S_{0}$ is simple. The definition of $w$ in 3.3) leads to

$$
w^{*} V_{0}\left(\beta_{i}\right)=e_{n}^{\top} B_{p, q}^{*} V_{0}\left(\beta_{i}\right)=e_{n}^{\top} B_{p}^{*} q^{\sharp}\left(C_{p^{\sharp}}\right) V_{0}\left(\beta_{i}\right)=e_{1}^{\top} q^{\sharp}\left(\beta_{i}\right) V_{0}\left(\beta_{i}\right)=q^{\sharp}\left(\beta_{i}\right) .
$$

Now the description of $S$ in Theorem 3.3 shows that $\widehat{v}_{i} \in S$. Hence, $\beta_{i}$ is an eigenvalue of $S$, and thus $S$ is not simple.

Finally, assume that $\alpha_{j} \in \sigma_{p}\left(A_{1}\right) \cap \sigma(q)$. Let $k_{j} \neq 0$ be the corresponding eigenvector of $A_{1}$ and let $\widehat{k}_{j}=\left\{k_{j}, \alpha_{j} k_{j}\right\}$. Then $\alpha_{j} \in \mathbb{R}, \Gamma_{1}^{0} \widehat{k}_{j}=0, p\left(\alpha_{j}\right) \neq 0$, and $\Gamma_{0}^{0} \widehat{k}_{j} \neq 0$. Now let $\widehat{u}_{j}=\left\{u_{j}, \alpha_{j} u_{j}\right\}$, where

$$
u_{j}=\frac{p\left(\alpha_{j}\right)}{\Gamma_{0}^{0} \widehat{k}_{j}} k_{j} \oplus\left(\begin{array}{c}
0 \\
V_{0}\left(\alpha_{j}\right)
\end{array}\right) .
$$


If $\operatorname{deg} p=\operatorname{deg} q$, then (3.3) and $\operatorname{Bez}(p, q)=-\operatorname{Bez}(q, p)=-B_{q} p\left(C_{q}\right)$ yield

$$
\bar{w}^{*} V_{0}\left(\alpha_{j}\right)=-e_{n}^{\top} B_{q} p\left(C_{q}\right) V_{0}\left(\alpha_{j}\right)=-e_{1}^{\top} q_{n} p\left(\alpha_{j}\right) V_{0}\left(\alpha_{j}\right)=-q_{n} p\left(\alpha_{j}\right) .
$$

Hence, in view of Theorem 3.3 $\widehat{u}_{j} \in S$. If $\operatorname{deg} p>\operatorname{deg} q=m$, then $q_{n}=0$ and

$$
\begin{aligned}
\bar{w}^{*} V_{0}\left(\alpha_{j}\right) & =e_{n}^{\top} B_{p, q} V_{0}\left(\alpha_{j}\right)=e_{n}^{\top} B_{p} q\left(C_{p}\right) V_{0}\left(\alpha_{j}\right)=e_{1}^{\top} q\left(C_{p}\right) V_{0}\left(\alpha_{j}\right) \\
& =q\left(e_{1}^{\top} C_{p}\right) V_{0}\left(\alpha_{j}\right)=\sum_{k=0}^{m} q_{k} e_{k+1}^{\top} V_{0}\left(\alpha_{j}\right)=q\left(\alpha_{j}\right)=0 .
\end{aligned}
$$

Moreover,

$$
C_{p} V_{0}\left(\alpha_{j}\right)+\Gamma_{0}^{0}\left(\frac{p\left(\alpha_{j}\right)}{\Gamma_{0}^{0} \widehat{k}_{j}} k_{j}\right) e_{n}=\left(\alpha_{j} V_{0}\left(\alpha_{j}\right)-p\left(\alpha_{j}\right) e_{n}\right)+p\left(\alpha_{j}\right) e_{n}=\alpha_{j} V_{0}\left(\alpha_{j}\right) .
$$

The description of $S$ in Theorem 3.3 again shows that $\widehat{u}_{j} \in S$. In both cases $\alpha_{j}$ is an eigenvalue of $S$, and thus $S$ is not simple. The case $\operatorname{deg} p<\operatorname{deg} q$ is obtained by changing the roles of $A_{0}$ and $A_{1}$ (cf. Corollary 3.2).

\section{REFERENCES}

[1] D. Alpay, A. Dijksma, J. Rovnyak, and H.S.V. de Snoo, Schur functions, operator colligations, and reproducing kernel Pontryagin spaces, Oper. Theory: Adv. Appl., 96, Birkhäuser Verlag, Basel, 1997. MR 2000a:47024

[2] V. Derkach, "On generalized resolvents of Hermitian relations", J. Math. Sciences, 97 (1999) No. 5, 4420-4460. MR 2001c:47042

[3] V.A. Derkach, S. Hassi, M.M. Malamud, and H.S.V. de Snoo, "Generalized resolvents of symmetric operators and admissibility", Methods of Functional Analysis and Topology, 6 (2000), 24-55. MR 2003b:47042

[4] V.A. Derkach, S. Hassi, and H.S.V. de Snoo, "Operator models associated with Kac subclasses of generalized Nevanlinna functions", Methods of Functional Analysis and Topology, 5 (1999), 65-87. MR 2001f: 47063

[5] V.A. Derkach, S. Hassi, and H.S.V. de Snoo, "Operator models associated with singular perturbations of selfadjoint operators", Methods of Functional Analysis and Topology, 7 (2001), 1-21. MR 2002m:47017

[6] V.A. Derkach, S. Hassi, and H.S.V. de Snoo, "Rank one perturbations in a Pontryagin space with one negative square", J. Funct. Anal., 188 (2002), 317-349. MR 2002k:47030

[7] V.A. Derkach, S. Hassi, and H.S.V. de Snoo, "Singular perturbations of selfadjoint operators", Mathematical Physics, Analysis and Geometry (to appear).

[8] V. Derkach and M. Malamud, "Generalized resolvents and the boundary value problems for hermitian operators with gaps", J. Funct. Anal., 95 (1991), 1-95. MR 93d:47046

[9] V. Derkach and M. Malamud, "The extension theory of hermitian operators and the moment problem", J. Math. Sciences, 73 (1995), 141-242. MR 95m:47009

[10] A. Dijksma, H. Langer, A. Luger, and Yu. Shondin, "A factorization result for generalized Nevanlinna functions of the class $\mathbf{N}_{\kappa}$ ", Integral Equations Operator Theory, 36 (2000), 121-125. MR 2000i: 47027

[11] A. Dijksma, H. Langer, Yu. Shondin, and C. Zeinstra, "Self-adjoint operators with inner singularities and Pontryagin spaces", Oper. Theory Adv. Appl., 117 (2000), 105-176. MR 2001f:47039

[12] A.Dijksma, H. Langer, H.S.V. de Snoo, "Symmetric Sturm-Liouville operators with eigenvalue depending boundary conditions", Canadian Math. Society, Conference Proceedings, 8 (1987), 87-116. MR 88m:34022

[13] V.I. Gorbachuk and M.L. Gorbachuk, Boundary value problems for operator differential equations, Naukova Dumka, Kiev, 1984 (Russian) (English translation: Kluwer Academic Publishers, Dordrecht, Boston, London, 1990). MR 92m:34133

[14] P. Jonas and H. Langer, "A model for $\pi_{1}$-spaces and a special linear pencil", Integral Equations Operator Theory, 8 (1985), 13-35. MR 86g:47047 
[15] P. Jonas, H. Langer, and B. Textorius, "Models and unitary equivalence of cyclic selfadjoint operators in Pontrjagin space", Oper. Theory Adv. Appl., 59 (1992), 252-284. MR 94j:47052

[16] M.G. Kreĭn and H. Langer, "Über die $Q$-Function eines $\pi$-hermiteschen Operators in Raume $\Pi_{\kappa} "$, Acta. Sci. Math. (Szeged), 34 (1973), 191-230. MR 47:7504

[17] M.G. Krĕn and H. Langer, "Über einige Fortsetzungsprobleme, die eng mit der Theorie hermitescher Operatoren in Raume $\Pi_{\kappa}$ zusammenhangen. I. Einige Funktionklassen und ihre Darstellungen", Math. Nachr., 77 (1977), 187-236. MR 57:1173

[18] M.G. Kreĭn and H. Langer, "Some propositions on analytic matrix functions related to the theory of operators in the space $\Pi_{\kappa}$ ", Acta Sci. Math. (Szeged), 43 (1981), 181-205. MR 82i: 47053

[19] P. Lancaster and M. Tismenetsky, The theory of matrices, Second edition. Academic Press, Inc., Orlando, FL, 1985. MR 87a:15001

[20] E.M. Russakovskiı̌, "An operator treatment of a boundary value problem with a spectral parameter that occurs polynomially in the boundary conditions", Functional Anal. Appl., 9 (1975), 358-359. MR 54:3505

[21] P. Sorjonen, "Pontryaginräume mit einem reproduzierenden Kern", Ann. Acad. Sci. Fenn. A.I. Math., 594, (1975). MR 53:8875

Department of Mathematics, Donetsk National University, Universitetskaya Str. 24, 83055 Donetsk, Ukraine

Current address: Department of Mathematics, Western Washington University, Bellingham, Washington 98225

E-mail address: derkacv@cc.wwu.edu

Department of Mathematics and Statistics, University of VaAsa, PL 700, 65101 Vaasa, FinLAND

E-mail address: sha@uwasa.fi 